\title{
Re: Osteoma-like melorheostosis: a rare type of skeletal dysplasia depicted on FDG PET/CT
}

\author{
Michael J. Klein ${ }^{1}$ (D) \\ Received: 31 July 2019 /Revised: 31 July 2019 / Accepted: 3 September 2019/Published online: 8 November 2019 \\ (C) ISS 2019
}

\section{To the Editor}

I am writing with regard to the manuscript published in the August edition of Skeletal Radiology entitled "Osteoma-like melorheostosis: a rare type of skeletal dysplasia depicted on FDG PET/CT." by Abi-Ghanem, Asmar, Boulos, and Muwakkit. This paper is well written and while it has a good introduction and an interesting short discussion of melorheostosis, I do not believe that the histological findings illustrated support that diagnosis.

The sclerosing skeletal dysplasias are almost always diagnosed using radiographic imaging and very seldom are biopsied. When they are biopsied, the histological features are almost never actually diagnostic, but they are usually confirmatory in the sense that they show nothing that would negate the radiologist's impression. The preceding statements are particularly true for cases of melorheostosis, which is seen many more times by skeletal radiologists than by skeletal pathologists. Biopsies of melorheostosis are performed when the imaging features are compatible with the diagnosis but are not absolutely diagnostic. The "confirmatory" histology of such cases demonstrates compact bone, mainly of lamellar fiber architecture, but sometimes mixed with small quantities of woven bone, in which there is an irregularity in the relationship of the osteons. In that sense, the term "osteoma-like melorheostosis" is completely redundant because most osteomas are composed of compact bone having very similar structure to melorheostosis. If there are actual trabeculae formed, these tend to become compact and form Haversian canals and the osseous lesions are almost completely devoid of stroma.

In the current paper, the bone formed consists of thick trabeculae of woven bone separated by a fairly cellular fibrovascular stroma. While there are a few structures resembling

Michael J. Klein

kleinm@hss.edu

1 Pathologist in Chief Emeritus, Hospital for Special Surgery, 535 East 70th Street, New York, NY 10021, USA
Haversian canals in these trabeculae, they may actually be part of the cortex given the localization of the process and in any case do not dominate the histology. If this lesion had been thought to be melorheostosis on the imaging studies, that notion should have been dispelled by these histological features. Despite the rather unusual imaging features mentioned in the publication, the histological features point rather strongly toward fibrous dysplasia. While the PET/CT data do show avid uptake roughly corresponding to an L5 dermatomal distribution, the diagnosis of melorheostosis cannot be made if it is disproven by the histological findings. It is possible to demonstrate that the lesion is fibrous dysplasia by searching for activating GS mutations (since these are demonstrable in about $70 \%$ of cases of fibrous dysplasia), but only if the lesion has been decalcified in neutral EDTA or some other decalcification method that is not acid based.

While it is true that cortical thinning and disruption are not generally part of fibrous dysplasia, the extension of fibrous dysplasia into soft tissues has been well described [1]. While the authors" description of "dense irregular trabeculae of woven bone devoid of osteoblastic rimming and separated by a fibroblastic stroma" is a completely accurate description of their figure 2, their reference 10 , which they state supports the diagnosis, illustrates highly remodeled and disorganized compact bone without a significant fibrous stroma [2] and illustrates a completely different entity than the one depicted in the current paper.

\section{References}

1. Kashima TG, Gamage NM, Ye H, et al. Locally aggressive fibrous dysplasia. Virchows Arch. 2013;463:79-84.

2. Hephzibah J, Theodore B, Oommen R, Cherian V, Eapen A, Deepti A. melorheostosis - scintigraphic and hostopathological features: a case report. Internet J Orthop Surg. 2006;4(2).

Publisher's note Springer Nature remains neutral with regard to jurisdictional claims in published maps and institutional affiliations. 